\title{
Vitamin D Status, Gender Differences, and Cardiometabolic Health Disparities
}

\author{
Dharambir K. Sanghera ${ }^{a, d-f}$ Bishwa R. Sapkota ${ }^{a, f}$ Christopher E. Aston ${ }^{b}$ \\ Piers R. Blackett ${ }^{c, f}$ \\ a Department of Pediatrics, Section of Genetics, b Section of Developmental and Behavioral Pediatrics, cSection of \\ Endocrinology, ${ }^{\mathrm{d}}$ Oklahoma Center for Neuroscience, ${ }^{e}$ Department of Pharmaceutical Sciences, ${ }^{\mathrm{f}} \mathrm{Harold} \mathrm{Hamm}$ \\ Diabetes Center, University of Oklahoma Health Sciences Center, Oklahoma City, OK, USA
}

\section{Keywords}

$25(\mathrm{OH})$ vitamin D - Type 2 diabetes · Cardiometabolic disease risk · Gender difference · Asian Indians

\begin{abstract}
Background: Vitamin D deficiency is an unrecognized epidemic found in India and also worldwide. Despite the high prevalence of diabetes among Indians, there is a paucity of data showing the relationship between vitamin $D$ status and cardiometabolic disparities. In this study, we have examined the relationship between vitamin $\mathrm{D}$ and cardiometabolic traits in a population from India. Methods: Circulating 25(OH) $D$ levels were measured in 3,879 participants from the Asian Indian Diabetic Heart Study using ELISA kits. Results: Vitamin D levels were significantly reduced $(p<0.0001)$ in both men and women with obesity. However, compared to women, serum vitamin D was consistently lower in men $(p<0.02)$, irrespective of the presence of obesity and type 2 diabetes. Multivariate regression revealed strong interaction of vitamin D with body mass index that resulted in increased fasting glucose $(p=0.001)$ and reduced homeostasis model assessment of $\beta$-cell function (HOMA-B; $p=0.01$ ) in normoglycemic individuals. However, in gender-stratified analysis, this association was restricted to men for both fasting glu-
\end{abstract}

cose $\left(p=2.4 \times 10^{-4}\right)$ and HOMA-B $(p=0.001)$. Conclusions: Our findings suggest that vitamin $D$ deficiency may significantly enhance the risk of cardiometabolic disease among Asian Indians. Future randomized trials and genetic studies are expected to clarify the underlying mechanisms for gender differences in vitamin D deficiency, and whether vitamin D-driven improvement in testosterone may contribute to beneficial cardiometabolic outcomes in men.

두 2017 S. Karger AG, Base

\section{Introduction}

Vitamin D deficiency is an under-recognized epidemic throughout the world, and over one billion people globally have insufficient or deficient vitamin D levels [1]. Vitamin $\mathrm{D}$ is a prohormone and having sufficient plasma vitamin D levels is considered a marker of "good" health. Apart from the well-established role of vitamin D in maintaining good skeletal health (including osteoporosis, fractures, calcium, phosphorous, and bone metabolism), vitamin D deficiency is also associated with increased risk of obesity, diabetes, cardiovascular disorders (CVDs), autoimmune-, infectious-, and neuro-degenerative diseases including Alzheimer's and Parkinson's disease [2, 3].

\section{KARGER}

(c) 2017 S. Karger AG, Basel

E-Mail karger@karger.com

www.karger.com/anm
Dharambir K. Sanghera, PhD, FAHA

Department of Pediatrics, College of Medicine

University of Oklahoma Health Sciences Center

940 Stanton L. Young Blvd., Rm 317 BMSB, Oklahoma City, OK 73104 (USA)

E-Mail Dharambir-sanghera@ouhsc.edu 
Although mounting evidence has suggested that the association of low vitamin $\mathrm{D}$ with non-skeletal conditions may have widespread health implications, these findings still need to be confirmed by carefully designed observational studies [4]. Risk factors, such as skin pigmentation, living at higher latitudes (above $35^{\circ} \mathrm{N}$ or below $35^{\circ} \mathrm{S}$ ), excessive use of sunscreen, completely covering the body with clothes, sedentary behaviors, tendency toward staying indoors all the time, obesity, body weight/body mass index (BMI), old age, chronic disease, atmospheric pollution, and genetics, leading to vitamin $\mathrm{D}$ deficiency primarily involve inadequate exposure to sunlight [3]. The presence of vitamin $\mathrm{D}$ receptor (VDR) in most body tissues indicates its broader physiological role beyond calcium and bone metabolism [5].

Vitamin D deficiency is an epidemic in India affecting $50-90 \%$ of the population with varying degrees of insufficiency [6]. India is located near the equatorial region and the climate in India and other South Asian countries is sunny throughout the year, thereby creating ample possibilities of exposure to sunlight. However, due to the dark skin pigmentation, people living in Indian subcontinents require double amount of UVB exposure to produce the same amount of vitamin D as compared to white Europeans [7]. A high prevalence of vitamin D deficiency is reported across the country among people in all age groups, both genders, lower to upper socio-economic status [8], urban and rural populations $[9,10]$, pregnant women and their new born [11], postmenopausal women [12], and young healthy adults [13]. However, a majority of these studies comprise a very small number of study participants (ranging from 12 to 150$)[14,15]$. Despite the high prevalence of cardiometabolic disease and vitamin D deficiency in India, there is paucity of data showing the relationship between vitamin D status and cardiometabolic traits in the Indian population.

Accumulating evidence from animal and human studies suggest that hypovitaminosis D may contribute to pancreatic beta cell dysfunction and insulin resistance, and hence to type 2 diabetes (T2D) [16]. Currently, 62.4 million people in India have T2D and 77.2 million have prediabetes [17], accounting for the highest prevalence after China [18]. Vitamin D levels are easily determined by measuring the level of circulating calcidiol $(25[\mathrm{OH}] \mathrm{D})$ in blood, which depends on exogenous supply through dietary sources, endogenous production in the skin, and the overall activities of vitamin D metabolic enzymes. We have previously reported a very high prevalence of vitamin $\mathrm{D}$ deficiency among 1,765 individuals (887 T2D patients and 878 controls) from Punjab, India. Vitamin D deficiency was found to be increased in proportion to T2D, obesity, and
CVD risk factors [15]. To gain insight into the effects of vitamin D deficiency present within the population, in this study, we report the association of gender differences in serum vitamin D deficiency with increased risk for cardiometabolic traits in our extended cohort comprised of 3,879 Asian Indians (2,198 T2D and 1,681 controls). In this study, we have also included UVB index data to adjust for the confounding effects of seasonal changes that may influence vitamin D concentrations. To our knowledge, this is the first comprehensive study on Asian Indians reporting the role of vitamin D status on cardiometabolic health with possible gender-specific health implications.

\section{Experimental Methods}

\section{Study Population}

Participants for this study were recruited as part of the Asian Indian Diabetic Heart Study (AIDHS)/Sikh Diabetes Study (SDS) $[19,20]$. The AIDHS is a population-based, cross-sectional study originally aimed to investigate the genetic and environmental factors responsible for a high prevalence of metabolic syndrome, T2D, and early cardiovascular disease in Punjabi Sikhs. A total of 3,879 adult participants of age $>25$ years were analyzed in this study. Sikhs are strictly a nonsmoking population and $50 \%$ of participants were teetotalers and lifelong vegetarians. The diagnosis of T2D was made based on medical records for symptoms or use of medications or diabetes was defined on the basis of the measured fasting glucose levels following the American Diabetes Association guidelines [21]. The selection of controls was based on a fasting blood glucose $(\mathrm{FBG})<100.8 \mathrm{mg} / \mathrm{dL}$ or glucose during oral glucose tolerance test $<141.0 \mathrm{mg} / \mathrm{dL}$. Participants with impaired fasting glucose or impaired glucose tolerance were excluded from the study. Insulin was measured by radio-immuno assay (Diagnostic Products, Cypress, USA). Homeostasis model assessment for insulin resistance (HOMA-IR) was calculated using the formula, fasting insulin $(\mu \mathrm{IU} / \mathrm{mL}) \times$ fasting glucose $(\mathrm{mmol} / \mathrm{L}) / 22.5$ as described previously [22]. BMI was calculated using the formula, weight $(\mathrm{kg}) /$ height (meter) $)^{2}$. Waist and hip circumferences were measured with a tape at the abdomen and at the hip levels. The World Health Organization (WHO) has recommended lower BMI thresholds for Asian populations; therefore, obesity was defined using WHO's new guidelines [23]; participants with BMI $<23 \mathrm{~kg} / \mathrm{m}^{2}$ were classified as "normal weight," those with BMI between 23 and $27.5 \mathrm{~kg} / \mathrm{m}^{2}$ were classified as "overweight," and those with BMI $>27.5 \mathrm{~kg} / \mathrm{m}^{2}$ were classified as "obese." Details on assessing the levels of physical activity, smoking, alcohol consumption, and diet are described elsewhere $[19,20,24]$. In general, Sikhs do not smoke due to religious and cultural reasons, and about $50 \%$ of the study participants are lifelong vegetarians. Blood pressure (BP) was measured twice after a 5 min seated rest period. To remove the confounding effects of seasonal variation on vitamin D synthesis, UVB index data were collected for each recruitment site. The quantum of UVB rays (290-310 nm) received by each individual was computed from the publicly available data either in Tropospheric Emission Monitoring Internet Service (http://www.temis.nl/uvradiation/GOME/ stations.html) or in National Weather Service, (http://www.cpc.
Sanghera/Sapkota/Aston/Blackett 
ncep.noaa.gov/products/stratosphere/uv_index/uv) corresponding to the month and year of recruitment.

All blood samples were obtained at the baseline visit. All participants provided informed consent following procedures approved by Institutional Review Boards (IRBs). All AIDHS/SDS protocols and consent documents were reviewed and approved by the University of Oklahoma Health Sciences Center IRB as well as the Human Subject Protection (Ethics) Committees at the participating hospitals and institutes in India.

\section{5(OH) Vitamin D Measurements}

$25(\mathrm{OH}) \mathrm{D}$ levels were quantified on fasting serum using standard monoclonal antibody-based ELISA kits from ALPCO Diagnostics (Salem, NH, USA) as we reported previously $[15,25]$. Samples were run in duplicate following the manufacturer's instructions.

\section{Statistical Analysis}

Group descriptive statistics was expressed as mean \pm SD. Serum $25(\mathrm{OH}) \mathrm{D}$ levels were analyzed as a continuous variable after log transformation or as a categorical variable either as deficient $(<30 \mathrm{nmol} / \mathrm{L})$ or sufficient $(\geq 30 \mathrm{nmol} / \mathrm{L})$. Vitamin D status was classified as "deficient" and "sufficient" based on the recommendation of the Institute of Medicine, Food and Nutrition Board [26] and as described recently [27].

Variables with skewed distributions were log transformed (e.g., 25[OH]D, FBG, fasting insulin, and HOMA-IR). Independent $t$ tests were used to analyze the association between serum $25(\mathrm{OH})$ $\mathrm{D}$ and BMI (normal/overweight/obese groups) to evaluate the effect of vitamin D deficiency on quantitative traits. The classification of BMI was based on BMI cut points recommended by WHO Expert Consultation for Asian populations as described above [23]. The association of serum vitamin $\mathrm{D}$ with cardiomatabolic traits was evaluated by multivariate regression analysis performed separately on T2D cases and normoglycemic controls. In addition to age, gender, and BMI, UVB index was also included as covariates to remove the confounding effects of seasonal variation. Regression analysis was modeled for the interaction effect of BMI and serum vitamin D level on cardiometabolic traits including FBG, fasting insulin, HOMA-IR, HOMA-B, systolic BP, and diastolic BP using data from normoglycemic controls. Gender differences (male/female) for continuous traits (e.g., age, BMI) were analyzed using the Student $t$ test (Table 1 ) and for categorical variables, the vitamin D status (sufficient/deficient) was assessed using the $\chi^{2}$ test (Fig. 1). Bonferroni correction $p$ value for rejecting the null hypothesis for $(n)$ number of test at $\alpha=0.05$ was calculated using $(\alpha / n)$.

All analyses were performed using SPSS for windows statistical package (IBM SPSS Statistics for Windows, version 20.0; IBM Corp, Armonk, NY, USA).

\section{Results}

Clinical characteristics of the study population, stratified by T2D and vitamin D status, are summarized in Table 1 . Of the 3,879 individuals analyzed, $55 \%$ were men and $57 \%$ had T2D. The mean age of the participants was $51.9 \pm 13.2$ years. Vitamin D deficiency was highly prevalent in this population. In this study, a total of $58 \%$ men and $51 \%$ women participants were classified as having the deficient vitamin D status ( $<30 \mathrm{nmol} / \mathrm{L}$; Fig. 1$)$. The distribution of serum vitamin D level was significantly different between the men and women participants ( $p=$ $\left.1.1 \times 10^{-5}\right)$.

Serum $25(\mathrm{OH}) \mathrm{D}$ levels were inversely correlated with obesity and were significantly reduced in overweight (BMI $\left.23-27.5 \mathrm{~kg} / \mathrm{m}^{2} ; p<0.0001\right)$ and obese (BMI $>27.5$ $\left.\mathrm{kg} / \mathrm{m}^{2} ; p<0.001\right)$ individuals ( $p_{\text {trend }}<0.0001$; Fig. $2 \mathrm{a}$ ) in T2D and even among healthy normoglycemic controls (Fig. 2b). This association remained significant $(p<0.02)$ in male and female participants separately (Fig. 2b). Notably, mean 25(OH)D levels remained consistently reduced among male participants irrespective of obesity and T2D status (Table 1; Fig. 2a, b).

Further, we evaluated the association of vitamin D status on obesity and cardiometabolic traits using Pearson's correlations and multivariate linear regression analysis on normoglycemic individuals (Table 2; online suppl. Table 2; for all online suppl. material, see www.karger. com/doi/10.1159/000458765). The analysis was adjusted for the confounding effects of age and UVB index as appropriate. A highly significant association of serum vitamin D was mainly observed with BMI $(\beta=-0.56, p=$ $\left.1.2 \times 10^{-7}\right)$ in combined samples, and this association remained significant in both male $\left(\beta=-0.67, p=4.6 \times 10^{-6}\right)$ and female $(\beta=-0.42, p=0.007)$ participants; the strength of association was more pronounced in men. While a significant association of HOMA-IR with vitamin D was seen only in females $(p=0.008)$, only marginal significance was observed in HOMA-B. Taken together, the observed strong association of serum vitamin $\mathrm{D}$ with $\mathrm{BMI}$ in normoglycemic participants suggests that vitamin D deficiency and obesity may together have an additive effect for influencing the extent of cardiometabolic risk.

We, therefore, further performed interaction analyses to assess the contribution of serum vitamin $\mathrm{D}$ and obesity (BMI) in normoglycemic individuals for impacting other cardiomatabolic risk traits including FBG, fasting insulin, HOMA-IR, HOMA-B, and systolic and diastolic BP. Only FBG and HOMA-B showed significant association in vitamin $\mathrm{D}$ and $\mathrm{BMI}$ interaction analysis (online suppl. Table 1). As shown in Table 3, interaction of BMI*VITD significantly modulated FBG $(p=0.001)$ and HOMA-B $(p=0.01)$ in the combined analysis. Inclusion of gender in the interaction model only impacted FBG (gender*BMI*VITD: $p=0.014$ ) but not HOMA-B (Table 3; online suppl. Table 1). However, in gender-stratified analysis, the BMI*VITD interactions affected FBG level only in men $\left(p=2.4 \times 10^{-4}\right)$ but not in women $(p=$ 
Table 1. Clinical characteristics of the study population with statistical comparisons based on gender

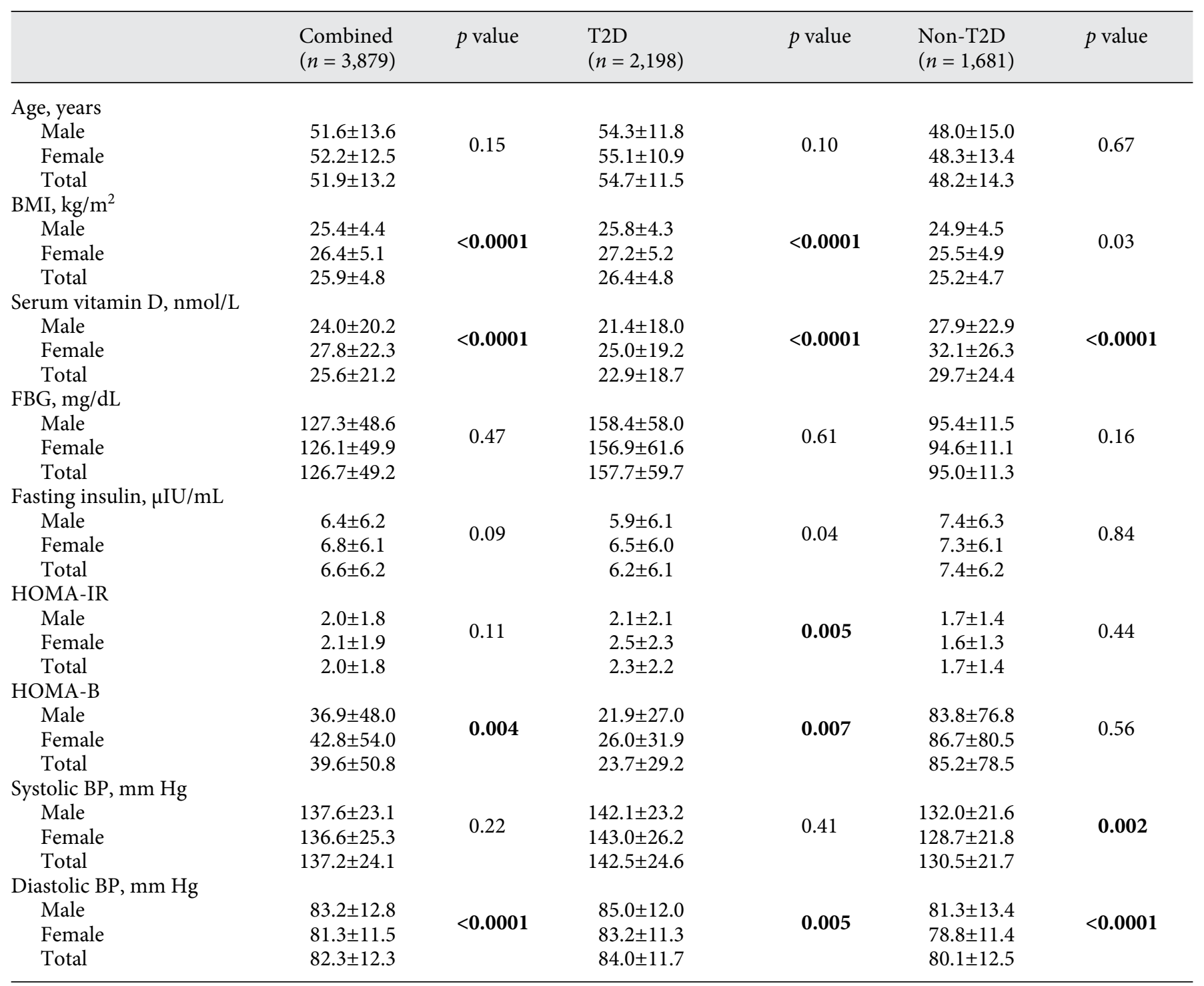

T2D, type 2 diabetes mellitus; non-T2D, normo-glycemic individuals; BMI, body mass index; FBG, fasting blood glucose; HOMA-IR, homeostasis model assessment for insulin resistance; HOMA-B, homeostasis model assessment of $\beta$-cell function; BP, blood pressure. Values are mean \pm SD. Differences between males and females were derived by independent $t$ test for the continuous variables to get the $p$ values, and significance $p$ value (male vs. female) after Bonferroni correction $(p=0.025)$ are in boldface.

0.990). The interaction $p$ values remained significant even when age was included in the model. Likewise, in multivariate interaction analysis for HOMA-B, a significant impact due to BMI*VITD interaction was observed only in men $(p=0.001)$ but not in women $(p=0.376$; Table 3$)$. Taken together, cardiometabolic risk traits including FBG and HOMA-B level were modulated by the BMI and serum vitamin $\mathrm{D}$ interactions, and this effect was mainly confined to men among the normoglycemic individuals.

\section{Discussion}

The relationship between obesity and $25(\mathrm{OH}) \mathrm{D}$ has been consistently reported and well established $[15,28-$ 30]. Our study confirms a strong inverse association of serum $25(\mathrm{OH}) \mathrm{D}$ with $\mathrm{BMI}$, and this inverse association with BMI remained consistently significant in both men and women when analyzed separately (Fig. 2). However, in gender-separated analysis in each BMI subgroup (normal/ 
Table 2. Multivariate linear regression analysis showing the association of serum vitamin $\mathrm{D}(25[\mathrm{OH}] \mathrm{D})$ level with cardiometabolic traits in normo-glycemic individuals

\begin{tabular}{|c|c|c|c|c|c|c|c|c|c|c|c|c|}
\hline Traits & \multicolumn{4}{|c|}{ Combined* } & \multicolumn{4}{|l|}{ Male $^{\wedge}$} & \multicolumn{4}{|c|}{ Female } \\
\hline $\mathrm{BMI}, \mathrm{kg} / \mathrm{m}^{2 \$}$ & 0.014 & $1.3 \mathrm{E}-06$ & $-0.56 \pm 0.11$ & $1.2 \mathrm{E}-07$ & 0.024 & $2.2 \mathrm{E}-06$ & $-0.67 \pm 0.15$ & $4.6 \mathrm{E}-06$ & 0.008 & 0.02 & $-0.42 \pm 0.16$ & 0.007 \\
\hline FBG, $\mathrm{mg} / \mathrm{dL}$ & 0.003 & 0.05 & $0.31 \pm 0.18$ & 0.08 & 0.006 & 0.03 & $0.46 \pm 0.24$ & 0.05 & 0.001 & 0.47 & $0.13 \pm 0.27$ & 0.62 \\
\hline Fasting insulin, $\mu \mathrm{IU} / \mathrm{mL}$ & 0.000 & 0.81 & $0.004 \pm 0.03$ & 0.90 & 0.003 & 0.23 & $-0.04 \pm 0.04$ & 0.36 & 0.002 & 0.34 & $0.05 \pm 0.04$ & 0.24 \\
\hline HOMA-IR & 0.002 & 0.12 & $0.06 \pm 0.03$ & 0.07 & 0.000 & 0.87 & $0.002 \pm 0.04$ & 0.97 & 0.014 & 0.009 & $0.13 \pm 0.05$ & 0.008 \\
\hline HOMA-B & 0.000 & 0.50 & $0.03 \pm 0.03$ & 0.35 & 0.002 & 0.27 & $-0.025 \pm 0.04$ & 0.51 & 0.008 & 0.05 & $0.08 \pm 0.04$ & 0.04 \\
\hline
\end{tabular}

BMI, body mass index; BP, blood pressure; FBG, fasting blood glucose; HOMA-IR, homeostasis model assessment for insulin resistance; HOMA-B, homeostasis model assessment of $\beta$-cell function; $\mathrm{R}^{2}, \mathrm{R}$-squared for each model and their $p$ values, $\mathrm{B} \pm \mathrm{SE}=$ unstandardized coefficient with standard error and their $p$ values after adjustment with covariates; to remove the confounding effects of seasonal variation, UVB index were used as covariate for adjustment in addition to age, BMI, and gender where appropriate (covariates: ${ }^{*}$ adjusted for age, BMI, UVB index, gender; ${ }^{\wedge}$ adjusted for age, BMI, UVB index; ${ }^{\$} \mathrm{BMI}$ was not used as covariate while analyzing BMI as a trait). Significant $p$ values are shown in boldface.

Fig. 1. Distribution of serum vitamin D concentrations in gender-stratified sample of a total of 3,879 study participants of the AIDHS/SDS. Pie graphs indicate percentage of vitamin $\mathrm{D}$ levels in dichotomous models: sufficient $(\geq 30 \mathrm{nmol} / \mathrm{L})$ and deficient $(<30 \mathrm{nmol} / \mathrm{L})$. Distribution of serum vitamin $\mathrm{D}$ concentrations was assessed by $2 \times 2$ chi-squared test $\left(\chi^{2}=19.3, p=1.1 \times\right.$ $\left.10^{-5}\right)$ in male and female participants.

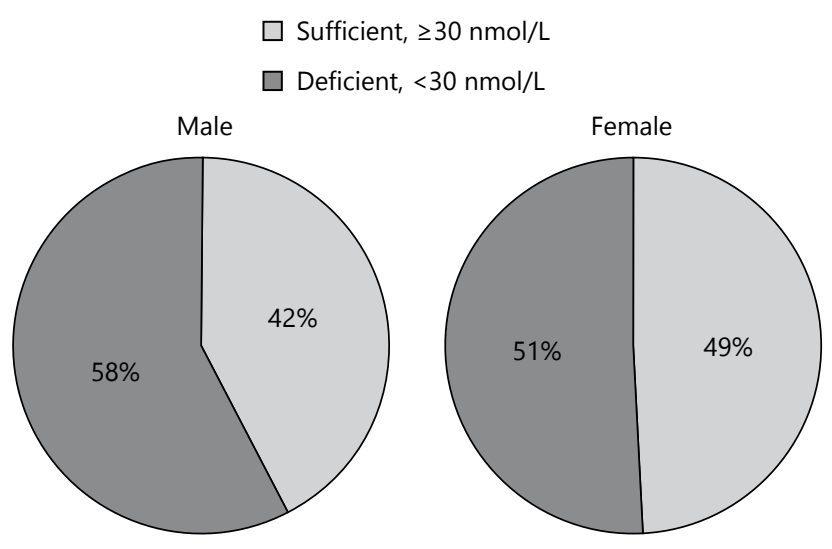

overweight/obese), vitamin D levels remained significantly reduced in men when compared to women, irrespective of obesity and T2D. Vitamin D is a fat-soluble hormone and its absorption is enhanced in obesity. Previously it was thought that obesity can lead to the sequestration of vitamin $\mathrm{D}$ in adipose tissue, thus reducing its bioavailability [31]. However, recent studies by Drincic et al. [32] could not find any evidence of sequestration of supplemental or endogenous $25(\mathrm{OH}) \mathrm{D}$ in fat cells. Their findings suggested that the dosing for vitamin $\mathrm{D}$ in obese patients should be adjusted according to body size and not according to BMI to achieve desired serum $25(\mathrm{OH}) \mathrm{D}$ concentrations [33]. Arguably, hyperparathyroidism, secondary to hypovitaminosis $\mathrm{D}$, augmented by obesity, could also be responsible for the observed association with obesity [34]. Largely, the obese people with T2D and a CVD risk profile are generally less active and may be less exposed to sunlight. Therefore, the possibility of reverse causation of vitamin D deficiency among the obese could also not be ruled out. Indeed, a large study on 42,024 individuals reported a causal link between vitamin D deficiency and obesity using a bi-directional genetic approach - the Mendelian Randomization - using 12 BMI-related single nucleotide polymorphisms. Their findings suggested that higher BMI leads to lower $25(\mathrm{OH}) \mathrm{D}$, whereas the contribution of lower $25(\mathrm{OH})$ D levels for increasing obesity would likely be small [35].

Also, in our study, the fasting glucose levels were increased with vitamin $\mathrm{D}$ deficiency in patients with $\mathrm{T} 2 \mathrm{D}$, but a significant increase was not observed in healthy normo-glycemic controls (online suppl. Table 1). In contrast 
Table 3. Multivariate interaction model on the association of serum vitamin D and obesity on metabolic traits in normo-glycemic individuals

\begin{tabular}{|c|c|c|c|c|c|c|}
\hline Parameters & \multicolumn{2}{|l|}{ Combined } & \multicolumn{2}{|l|}{ Male } & \multicolumn{2}{|l|}{ Female } \\
\hline Gender & $-1.134 \pm 0.493$ & 0.022 & - & - & & \\
\hline Age & $-0.000 \pm 0.001$ & 0.751 & $0.001 \pm 0.000$ & 0.060 & $0.001 \pm 0.000$ & 1.1E-04 \\
\hline BMI & $-0.625 \pm 0.225$ & 0.005 & $-0.276 \pm 0.095$ & 0.004 & $0.074 \pm 0.120$ & 0.539 \\
\hline Gender*BMI & $0.350 \pm 0.154$ & 0.023 & - & - & - & - \\
\hline Gender*vitamin D & $0.315 \pm 0.136$ & 0.021 & - & - & - & - \\
\hline $\mathrm{BMI}^{*}$ vitamin D & $0.203 \pm 0.064$ & 0.001 & $0.101 \pm 0.027$ & $2.4 \mathrm{E}-04^{\$}$ & $-0.0004 \pm 0.033$ & 0.990 \\
\hline Gender*BMI*vitamin D & $-0.102 \pm 0.043$ & 0.017 & - & - & - & - \\
\hline \multicolumn{7}{|l|}{$H O M A-B$} \\
\hline Gender & $7.450 \pm 4.365$ & 0.088 & - & - & - & - \\
\hline Gender*BMI & $-2.429 \pm 1.359$ & 0.074 & - & - & - & - \\
\hline Gender*vitamin D & $-1.832 \pm 1.212$ & 0.131 & - & - & - & - \\
\hline $\mathrm{BMI}^{*}$ vitamin D & $-1.473 \pm 0.593$ & 0.013 & $-0.859 \pm 0.260$ & $0.001^{\$}$ & $-0.246 \pm 0.277$ & 0.376 \\
\hline Gender*BMI*vitamin D & $0.613 \pm 0.380$ & 0.106 & - & - & - & - \\
\hline
\end{tabular}

FBG, fasting blood glucose; BMI, body mass index; HOMA-B, homeostasis model assessment of $\beta$-cell function. Significance $p$ value are in boldface.

$\$ p$ values remain significant even after adjustment with alcohol consumption in male participants.

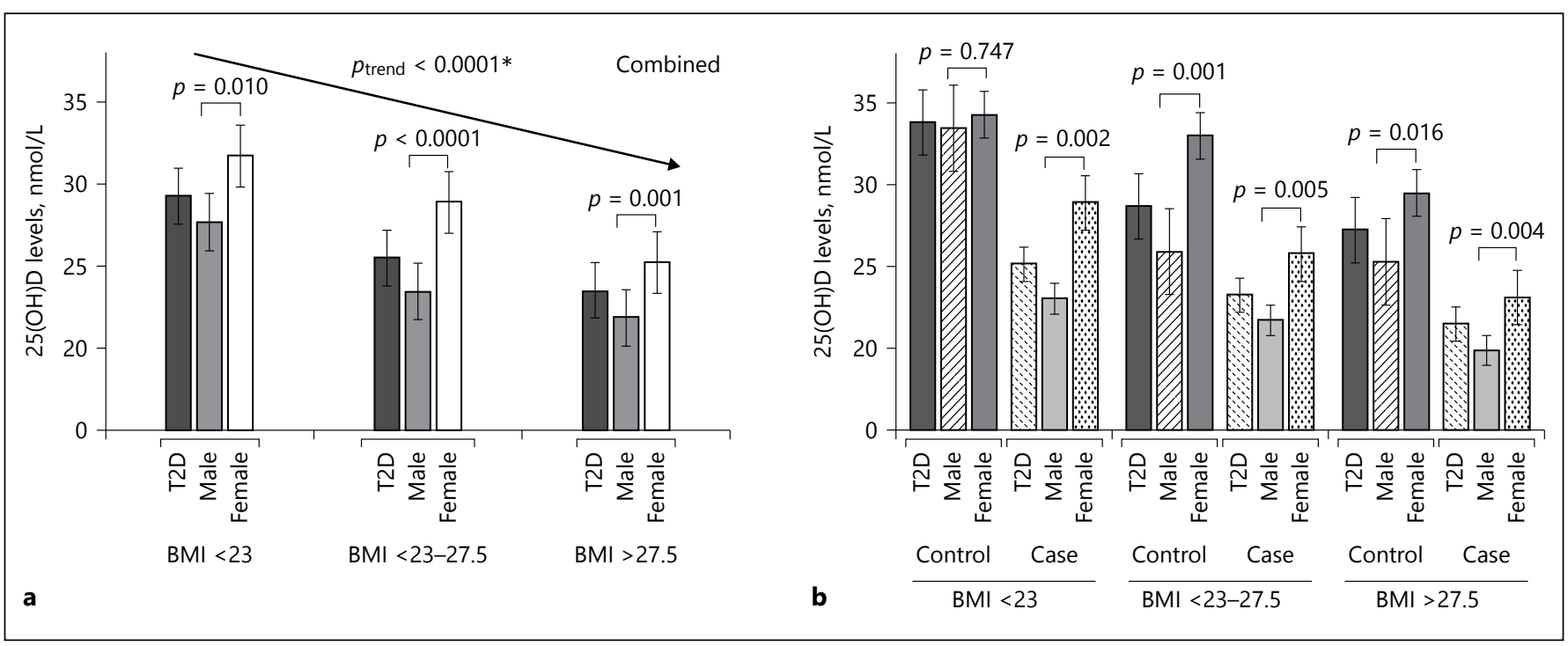

Fig. 2. a, b Association of BMI with serum vitamin $\mathrm{D}[25(\mathrm{OH}) \mathrm{D}]$ levels in participants stratified by gender (a) and T2D status (b). T2D, type 2 diabetes mellitus. The $p$ value indicates the mean com- parison of serum vitamin D levels in male and female in each category. ${ }^{*} p_{\text {trend }}$ value on comparison to different BMI categories in the entire cohort. 
to the NHANES data for 2005-2010, which showed that men tend to have a higher FBG [36], in AIDHS, we observed that the FBG levels were comparable between nondiabetic healthy men $(95.4 \mathrm{mg} / \mathrm{dL})$ and women $(94.6 \mathrm{mg} /$ $\mathrm{dL}$ ) when the sample was not stratified by vitamin D status. However, FBG was significantly increased and HOMA-B was significantly decreased in obese individuals with vitamin $\mathrm{D}$ deficiency, as revealed in interaction analysis performed in normoglycemic controls (Table 3). These data are in line with the existing evidence that vitamin D plays a critical role in insulin secretion and insulin action [16, 37], and that vitamin D status may influence the progression to risk for cardiomatabolic disease including T2D through BMI [37]. Notably, in gender-specific analysis, this observation was restricted to male participants among normoglycemic individuals (Table 3). Although the inverse association of $25(\mathrm{OH}) \mathrm{D}$ with fasting glucose and insulin has been shown earlier in non-Hispanic whites and Mexican Americans in NHANES, differences according to gender were not reported [38]. A number of earlier studies have reported the direct or indirect effect of vitamin D to insulin response either through the receptors or vitamin $\mathrm{D}$ concentrations influencing the gene function in the pathway for insulin secretion [39-43]. Sex-specific confounders, such as endogenous testosterone secretion, may account for differences in the effect of low 25(OH)D in males and females, since vitamin $\mathrm{D}$ is known to effect androgen synthesis in testicular cells [44], and possibly ovarian androgens with high testosterone levels in women [45]. Several earlier studies have reported an increase in $25(\mathrm{OH}) \mathrm{D}$ levels in women who are on oral contraceptives [46-48]. Also, earlier studies have independently confirmed the positive association of vitamin $\mathrm{D}$ and testosterone levels $[49,50]$ and vitamin D supplementation increases testosterone levels in men [49]. To our knowledge, a vast majority of our participants (particularly women) were not on hormonal therapy and this finding was based on their clinical reports or self-reported medication details. Importantly, VDRs and vitamin D metabolizing enzymes (CYP2R1, CYP27B1, and CYP24A1) are widely expressed in human testis [51, 52]. The VDR knockout mice have exhibited significant gonadal insufficiency and decreased sperm count and motility $[51,53]$. Gender difference in the skeletal phenotype was also displayed in male mice lacking VDR gene [54]. Also, the low vitamin D status is associated with increased diabetes risk in hypo gonadal men [55]. Our own study of telomere length revealed that Sikh men had significantly shorter telomere length compared to Sikh women, and shorter telomere length was associated with significantly increased risk of cardiomatabolic diseases

Impact of Gender Differences in Vitamin D Deficiency
[20]. The other possible explanation for gender-specific association could be the difference in alcohol intake between men and women. Indian women (Sikh) do not consume alcohol due to cultural and religious reasons. Prolonged alcohol consumption among men reduces the absorption and activation of vitamin D [56, 57]. Moreover, worldwide prevalence rates of CVD-related mortality are higher in men including Asian Indians $[55,58,59]$. Therefore, further studies will be needed to understand the implicit link between hypogonadism (specifically lower testosterones), vitamin D insufficiency, and cardiomatabolic disease outcomes in men.

We also observed the significant association of serum vitamin D level with obesity and cardiometabolic traits in an entire cohort; and an analysis based on T2D status revealed that the observed association was mostly mediated by diabetes. However, in normoglycemic participants, it appears that in vitamin $\mathrm{D}$ deficiency, interaction with obesity may further compound the total risk of cardiomatabolic diseases by increasing FBG by reduced beta cell function (HOMA-B). Oh et al. [60] found that the active form of vitamin D inhibits foam cell formation and suppresses macrophage cholesterol uptake in patients with T2D. Therefore, a direct protective effect of vitamin $\mathrm{D}$ on the improved beta cell function and endothelium is possible, since vitamin $\mathrm{D}$ deficiency has been associated with impaired beta cells and endothelial dysfunction [61].

Our study has both strengths and weaknesses. This is the first large comprehensive study reporting the role of serum vitamin $\mathrm{D}$ deficiency in cardiometabolic health in a population from South Asia. Furthermore, our data of $25(\mathrm{OH}) \mathrm{D}$ levels have been adjusted for seasonal changes that may influence vitamin $\mathrm{D}$ status during the winter months. Individuals on vitamin D supplementation were excluded from the study. Some limitations of our study include the case-control and cross-sectional study design and lack of data on testosterone and estrogen levels in the study participants. In summary, the results from our findings suggest that vitamin $\mathrm{D}$ deficiency may significantly enhance the risk of cardiometabolic disease in Asian Indians. Since Asian Indians, particularly men, are known to be susceptible to the metabolic syndrome, T2D, and early coronary artery disease $[19,22,62]$, vitamin D deficiency may put them at additional risk, making a strong case for early intervention, especially in obesity. Future randomized control trials and genetic studies would clarify the mechanism underlying the gender differences in vitamin $\mathrm{D}$ deficiency, and whether vitamin $\mathrm{D}$-driven improvement in testosterone may contribute to beneficial cardiomatabolic outcomes in men.

Ann Nutr Metab 2017;70:79-87 


\section{Acknowledgments}

This work was supported by NIH grants -R01DK082766 funded by the National Institute of Health and NOT-HG-11-009 funded by National Human Genome Research Institute (NHGRI) and Phen X Rising Consortium (NHGRI), and grants from the Oklahoma Center for Neuroscience, and Harold Hamm Diabetes Center. Technical support provided by Timothy Braun, Latonya Been, and Praveen Natt is duly acknowledged. Authors thank all the participants of AIDHS/SDS who made this study possible.

\section{Disclosure Statement}

The authors have no conflicts of interest to declare.

\section{Authorship}

Conceived and designed the experiments: D.K.S.; manuscript preparation: D.K.S. and B.R.S.; data analysis: B.R.S. and C.E.A. All authors read and provided their critical comments on the manuscript.

\section{References}

1 Holick MF: Vitamin D deficiency. N Engl J Med 2007;357:266-281.

2 Autier P, et al: Vitamin D status and ill health: a systematic review. Lancet Diabetes Endocrinol 2014;2:76-89.

3 Theodoratou E, et al: Vitamin D and multiple health outcomes: umbrella review of systematic reviews and meta-analyses of observational studies and randomised trials. BMJ 2014;348:g2035.

4 Khan $\mathrm{H}$, et al: Vitamin $\mathrm{D}$, type 2 diabetes and other metabolic outcomes: a systematic review and meta-analysis of prospective studies. Proc Nutr Soc 2013;72:89-97.

5 Mathieu C, et al: Vitamin D and diabetes. Diabetologia $2005 ; 48: 1247-1257$.

6 Harinarayan CV, Joshi SR: Vitamin D status in India - its implications and remedial measures. J Assoc Physicians India 2009;57:4048.

7 Lo CW, Paris PW, Holick MF: Indian and Pakistani immigrants have the same capacity as Caucasians to produce vitamin D in response to ultraviolet irradiation. Am J Clin Nutr 1986;44:683-685.

8 Puri S, et al: Vitamin D status of apparently healthy schoolgirls from two different socioeconomic strata in Delhi: relation to nutrition and lifestyle. Br J Nutr 2008;99:876-882.

9 Ramakrishnan S, et al: Vitamin D status and its seasonal variability in healthy young adults in an Asian Indian urban population. Endocr Pract 2011;17:185-191.

10 Goswami R, et al: Presence of $25(\mathrm{OH}) \mathrm{D}$ deficiency in a rural North Indian village despite abundant sunshine. J Assoc Physicians India 2008;56:755-757.

11 Marwaha RK, et al: Vitamin D status in pregnant Indian women across trimesters and different seasons and its correlation with neonatal serum 25-hydroxyvitamin D levels. Br J Nutr 2011;106:1383-1389.

12 Harinarayan CV: Prevalence of vitamin D insufficiency in postmenopausal south Indian women. Osteoporos Int 2005; 16:397-402.

13 Tandon N, et al: Bone mineral parameters in healthy young Indian adults with optimal vitamin D availability. Natl Med J India 2003; 16:298-302.
14 Marwaha RK, et al: Vitamin D status in healthy Indians aged 50 years and above. J Assoc Physicians India 2011;59:706-709.

15 Braun TR, et al: Vitamin D deficiency and cardio-metabolic risk in a North Indian community with highly prevalent type 2 diabetes. J Diabetes Metab 2012;3.

16 Chiu KC, et al: Hypovitaminosis D is associated with insulin resistance and beta cell dysfunction. Am J Clin Nutr 2004;79:820-825.

17 Anjana RM, et al: Prevalence of diabetes and prediabetes (impaired fasting glucose and/or impaired glucose tolerance) in urban and rural India: phase I results of the Indian Council of Medical Research-INdia DIABetes (ICMRINDIAB) study. Diabetologia 2011;54:30223027.

18 International Diabetes Federation: The International Diabetes Federation Diabetes Atlas Brussels, 2011.

19 Saxena R, et al: Genome-wide association study identifies a novel locus contributing to type 2 diabetes susceptibility in Sikhs of Punjabi origin from India. Diabetes 2013;62: 1746-1755.

20 Saxena R, et al: Genome-wide association study identifies variants in casein kinase II (CSNK2A2) to be associated with leukocyte telomere length in a Punjabi Sikh diabetic cohort. Circ Cardiovasc Genet 2014;7:287295.

21 American Diabetes Association: Diagnosis and classification of diabetes mellitus. Diabetes Care 2004;27(suppl 1):S5-S10.

22 Been LF, et al: Variants in KCNQ1 increase type II diabetes susceptibility in South Asians: a study of 3,310 subjects from India and the US. BMC Med Genet 2011;12:18.

23 WHO Expert Consultation: Appropriate body-mass index for Asian populations and its implications for policy and intervention strategies. Lancet 2004;363:157-163.

24 Sanghera DK, et al: The Khatri Sikh Diabetes Study (SDS): study design, methodology, sample collection, and initial results. Hum Biol 2006;78:43-63.

25 McCarty CA, et al: PhenX RISING: real world implementation and sharing of PhenX measures. BMC Med Genomics 2014;7:16.
26 Ross AC, et al: The 2011 report on dietary reference intakes for calcium and vitamin $\mathrm{D}$ from the Institute of Medicine: what clinicians need to know. J Clin Endocrinol Metab 2011;96:53-58.

27 Cashman KD, et al: Vitamin D deficiency in Europe: pandemic? Am J Clin Nutr 2016;103: 1033-1044.

28 Bell NH, et al: Evidence for alteration of the vitamin $\mathrm{D}$-endocrine system in obese subjects. J Clin Invest 1985;76:370-373.

29 Muscogiuri G, et al: 25-Hydroxyvitamin D concentration correlates with insulin-sensitivity and BMI in obesity. Obesity (Silver Spring) 2010;18:1906-1910.

30 Lenders CM, et al: Relation of body fat indexes to vitamin D status and deficiency among obese adolescents. Am J Clin Nutr 2009;90: 459-467.

31 Wortsman J, et al: Decreased bioavailability of vitamin D in obesity. Am J Clin Nutr 2000;72: 690-693.

32 Drincic A, et al: 25-Hydroxyvitamin D response to graded vitamin $\mathrm{D}_{3}$ supplementation among obese adults. J Clin Endocrinol Metab 2013;98:4845-4851.

33 Drincic AT, et al: Volumetric dilution, rather than sequestration best explains the low vitamin D status of obesity. Obesity (Silver Spring) 2012;20:1444-1448.

34 Parekh D, et al: Pilot study to evaluate the effect of short-term improvement in vitamin D status on glucose tolerance in patients with type 2 diabetes mellitus. Endocr Pract 2010; 16:600-608.

35 Vimaleswaran KS, et al: Causal relationship between obesity and vitamin $\mathrm{D}$ status: bi-directional Mendelian randomization analysis of multiple cohorts. PLoS Med 2013;10:e1001383.

36 Menke A, et al: Hemoglobin A1c, fasting plasma glucose, and 2-hour plasma glucose distributions in U.S. population subgroups: NHANES 2005-2010. Ann Epidemiol 2014; 24:83-89.

37 Abbasi F, et al: Low circulating 25-hydroxyvitamin D concentrations are associated with defects in insulin action and insulin secretion in persons with prediabetes. J Nutr 2015;145: 714-719. 
38 Christensen MH, Scragg RK: Consistent ethnic specific differences in diabetes risk and vitamin D status in the National Health and Nutrition Examination Surveys. J Steroid Biochem Mol Biol 2016;164:4-10.

39 Maestro B, et al: Transcriptional activation of the human insulin receptor gene by 1,25-dihydroxyvitamin $\mathrm{D}(3)$. Cell Biochem Funct 2002;20:227-232.

40 Mitri J, et al: Effects of vitamin D and calcium supplementation on pancreatic $\beta$ cell function, insulin sensitivity, and glycemia in adults at high risk of diabetes: the calcium and vitamin $\mathrm{D}$ for diabetes mellitus (CaDDM) randomized controlled trial. Am J Clin Nutr 2011;94:486-494.

41 Pittas AG, et al: The role of vitamin D and calcium in type 2 diabetes. A systematic review and meta-analysis. J Clin Endocrinol Metab 2007;92:2017-2029.

42 Berchtold MW, Brinkmeier H, Müntener M: Calcium ion in skeletal muscle: its crucial role for muscle function, plasticity, and disease. Physiol Rev 2000;80:1215-1265.

43 Hamilton B: Vitamin D and human skeletal muscle. Scand J Med Sci Sports 2010;20:182190.

44 Hofer D, et al: Testicular synthesis and vitamin D action. J Clin Endocrinol Metab 2014; 99:3766-3773.

45 Ding EL, et al: Sex differences of endogenous sex hormones and risk of type 2 diabetes: a systematic review and meta-analysis. JAMA 2006;295:1288-1299.
46 Harris SS, Dawson-Hughes B: The association of oral contraceptive use with plasma 25-hydroxyvitamin D levels. J Am Coll Nutr 1998; 17:282-284.

47 Harmon QE, Umbach DM, Baird DD: Use of estrogen-containing contraception is associated with increased concentrations of 25-hydroxy vitamin D. J Clin Endocrinol Metab 2016;101:3370-3377.

48 García-Bailo B, et al: Plasma 25-hydroxyvitamin $\mathrm{D}$, hormonal contraceptive use, and cardiometabolic disease risk in an ethnically diverse population of young adults. J Am Coll Nutr 2013;32:296-306.

49 Pilz S, et al: Effect of vitamin D supplementation on testosterone levels in men. Horm Metab Res 2011;43:223-225.

50 Wehr E, et al: Association of vitamin D status with serum androgen levels in men. Clin Endocrinol (Oxf) 2010;73:243-248.

51 Blomberg Jensen $M$, et al: Vitamin D receptor and vitamin $\mathrm{D}$ metabolizing enzymes are expressed in the human male reproductive tract. Hum Reprod 2010;25:1303-1311.

52 Aquila S, et al: Human male gamete endocrinology: 1alpha, 25-dihydroxyvitamin D3 $(1,25(\mathrm{OH}) 2 \mathrm{D} 3)$ regulates different aspects of human sperm biology and metabolism. Reprod Biol Endocrinol 2009;7:140.

53 Kwiecinski GG, Petrie GI, DeLuca HF: Vitamin $\mathrm{D}$ is necessary for reproductive functions of the male rat. J Nutr 1989;119:741-744.

54 Ryan JW, et al: Sex-related differences in the skeletal phenotype of aged vitamin $\mathrm{D}$ receptor global knockout mice. J Steroid Biochem Mol Biol 2016;164:361-368.

55 Oh JY, et al: Endogenous sex hormones and the development of type 2 diabetes in older men and women: the Rancho Bernardo study. Diabetes Care, 2002;25:55-60.

56 Laitinen K, et al: Deranged vitamin D metabolism but normal bone mineral density in Finnish noncirrhotic male alcoholics. Alcohol Clin Exp Res 1990;14:551-556.

57 Bjørneboe GE, et al: Effect of alcohol consumption on serum concentration of 25-hydroxyvitamin D3, retinol, and retinol-binding protein. Am J Clin Nutr 1986;44:678682.

58 Kalin MF, Zumoff B: Sex hormones and coronary disease: a review of the clinical studies. Steroids 1990;55:330-352.

59 Barrett-Connor E: Sex differences in coronary heart disease. Why are women so superior? The 1995 Ancel Keys Lecture. Circulation 1997;95:252-264.

$60 \mathrm{Oh} \mathrm{J}$, et al: $1,25(\mathrm{OH}) 2$ vitamin d inhibits foam cell formation and suppresses macrophage cholesterol uptake in patients with type 2 diabetes mellitus. Circulation 2009;120:687-698.

61 Ertek S, et al: 25-Hydroxy vitamin D levels and endothelial vasodilator function in normotensive women. Arch Med Sci 2012;8:4752.

62 Kooner JS, et al: Genome-wide association study in individuals of South Asian ancestry identifies six new type 2 diabetes susceptibility loci. Nat Genet 2011;43:984-989. 